\title{
Der Prozess der Entwicklung von Zentren am Beispiel des Darmkrebszentrums
}

\author{
The Development Process of Colon Cancer Centres
}

Autoren

Institute
M. Sahm ${ }^{1,3}$, S. Wesselmann ${ }^{2}$, R. Kube ${ }^{3}$, N. Schöffel ${ }^{4}$, M. Pross ${ }^{1}$, H. Lippert ${ }^{3,5}$, S. Kahl ${ }^{6}$

Die Institutsangaben sind am Ende des Beitrags gelistet
Schlüsselwörter

- kolorektales Karzinom

- evidenzbasierte Medizin

- Darmkrebszentrum

Key words

- colorectal carcinoma

evidence-based medicine

- colon cancer centre

\section{Zusammenfassung \\ $\nabla$}

Kolonkarzinome stellen unverändert einen der häufigsten malignen Tumoren in der westlichen Welt dar. Multizentrische Beobachtungsstudien haben wichtige Erkenntnisse über die flächendeckende Versorgungsqualität der Patienten in der klinischen Routine erbracht. Eine Qualitätssteigerung in der Behandlung ist durch eine weitere Verbesserung der diagnostischen und therapeutischen Maßnahmen im interdisziplinären Setting zu erreichen. Das Konzept der Darmkrebszentrumsbildung verfolgt eine Optimierung der objektivierbaren Behandlungsergebnisse durch die Strukturbildung einer interdisziplinär und transsektoral wirkenden Einheit, welche die gesamte Versorgungskette für den Patienten umfasst. Die Implementierung der aktuellen Erkenntnisse der evidenzbasierten Medizin in den klinischen Alltag, die Überprüfung der Anwendung der Leitlinieninhalte durch externe Fachexperten in Form eines Audits und das steuernde Korrigieren bei analysierten Defiziten im Behandlungsprozess stellen den Garant für eine kontinuierliche Weiterentwicklung dar.

\section{Hintergrund}

Bibliografie

DOI http://dx.doi.org/

10.1055/s-0032-1315115

Online-publiziert 31.10.2012

Zentralbl Chir 2013; 138:

33-37 (c) Georg Thieme

Verlag KG Stuttgart · New York .

ISSN 0044-409X

Korrespondenzadresse

PD Dr. Maik Sahm

Klinik für Chirurgie, Darm-

zentrum Treptow-Köpenick

DRK Kliniken Berlin | Köpenick

S.-Allende-Straße 2-8

12559 Berlin

Deutschland

m.sahm@drk-kliniken-berlin.de
Kolorektale Karzinome stellen in den Ländern der westlichen Welt einen der häufigsten malignen Tumoren dar. Geschlechtsunabhängig betrachtet, ist es in Deutschland die häufigste Krebserkrankung [1,2]. Die jährliche Neuerkrankungsrate wird mit 73000 Erkrankungen angegeben und ist fast gleich auf Männer und Frauen verteilt [2]. Entsprechend der ICD-10-Diagnosegruppen C18-C21 war die Neuerkrankungsrate in Deutschland im Jahr 2006 auf Platz 2 im internationalen Vergleich für altersspezifische Neuerkrankungen bei Männern und bei Frauen [2,3]. Damit bedingt das kolorektale Karzinom bei den Männern 16,2\% aller

\section{Abstract \\ $\nabla$}

Colon carcinomas are the most common malignant tumours in the Western world. Important findings about the overall quality of medical care have been reported in multi-centre observational studies. A quality enhancement of therapeutic care can be achieved by an additional increase in diagnostic and therapeutic measures in the interdisciplinary setting. The development of colon cancer centres improves the chance to objectively observe the results of medical care induced by the development of an interdisciplinary and crosssectoral unit that includes a comprehensive medical care for patients. The implementation of the current medical findings based on evidence in clinical routine, the inspection of the usage of guidelines by external specialists as part of an audit and the continuous correction of analysed deficits in the course of treatment guarantee a continuous improvement of service.
Krebserkrankungen und bei Frauen 17,5\% [2]. Die gruppen für das Jahr 2005 vom Statistischen Bundesamt Wiesbaden mit 13569 Männern und 13654 Frauen angegeben, was, bezogen auf alle Krebssterbefälle, einem Prozentsatz von 12,4\% bei den Männern und $14,3 \%$ bei den Frauen entspricht [4].

Dank der Fortschritte in der Diagnostik und Therapie haben sich die Überlebenschancen bei Patienten mit Kolonkarzinomen in Deutschland in den letzten Jahrzehnten verbessert. Vor allem in Anbetracht der hohen Anzahl von Neuerkrankungen bleibt die Herausforderung unvermindert bestehen, durch eine Optimierung der diagnosti- 
schen und therapeutischen Maßnahmen eine weitere Qualitätssteigerung in der flächendeckenden Behandlung der Patienten zu erreichen. Mit dem Konzept der Darmkrebszentrenbildung soll dieses Ziel mit einer Verbesserung der objektivierbaren Behandlungsergebnisse erreicht werden [5]. Neben der Bildung eines krankheitsspezifischen, umfassenden und kompetenten Netzwerks für den Patienten und seine Angehörigen wird durch die Bildung der Darmkrebszentren die Implementierung evidenzbasierter Inhalte der S3-Leitlinie „Kolorektales Karzinom“ in den klinischen Alltag gefordert und gefördert. Die Darmkrebszentren müssen im Rahmen der Zertifizierung nachweisen, dass sie ihre Patienten leitliniengetreu behandeln. Eventuelle Defizite bei der Umsetzung dieser Vorgaben werden kritisch analysiert. Diese werden interdisziplinär diskutiert und im Sinne eines klassischen Plan-Do-Check-Act-Zyklus mit steuernden Korrekturmaßnahmen beseitigt.

\section{Qualitätssicherung in der Chirurgie}

Den 1. Schritt zur Verbesserung der therapeutischen Ergebnisse in der Chirurgie stellt die Qualitätssicherung durch klinische Versorgungsforschung dar. Bereits Theodor Billroth (1829-1894) erkannte den Nutzen der Ergebnisdokumentation und Auswertung, welche eine Analyse der Therapieresultate erlaubte und eine Problemerkennung gestattete [6]. Die ergebnisorientierte Qualitätssicherung mit dem Ziel der Identifikation fehlerhafter Prozeduren und ihrer Vermeidung bei künftigen Patienten wurde vom Bostoner Chirurg Ernest Amory Codman (1869-1940) initiiert [7]. Er forderte die Ergebnisdokumentation der Krankenhäuser und den Vergleich untereinander und ging mit jährlichen Behandlungsberichten seiner Patienten voran [8,9]. Seine Empfehlungen sowie die von Abraham Flexner (1866-1959) führten in Amerika zur Entwicklung eines Zertifizierungsprogramms. Daraus entstand 1951 in Chicago die "Joint Commission on Accreditation of Healthcare Organizations“ als gemeinnützige Organisation zur Beratung und Bewertung von Krankenhäusern [10].

In Deutschland hat die Erstellung von Qualitätsanforderungen zur Versorgung von Patienten mit kolorektalen Karzinomen einen hohen Stellenwert und eine lange Tradition. Die Ergebnisse von Forschungsgruppen und Literaturanalysen zeigen, dass durch Therapie- und Strukturoptimierungen eine Verbesserung der chirurgischen und multimodalen Behandlungsqualität mit nachfolgender Prognoseverbesserung für den Patienten mit Kolon- und Rektumkarzinomen erreicht wird [11]. Für das Rektumkarzinom wurden 1995 erste Qualitätsindikatoren von einer Expertengruppe der Deutschen Krebsgesellschaft formuliert [12]. 2006 erfolgte eine Überprüfung und Aktualisierung der Qualitätsindikatoren durch die interdisziplinäre Arbeitsgruppe Workflow Rektumkarzinom II mit Publikation des Konsenses [12]. Um den Stellenwert des Chirurgen als Prognosefaktor bei der Versorgung von Kolon- und Rektumkarzinomen festzulegen, publizierte die Forschungsgruppe Onkologie Gastrointestinaler Tumoren (FOGT) 2009 Qualitätsparameter für die multimodale Therapie von Kolon- und Rektumkarzinomen [11]. Als zentrales Problem wurden die Varianz der onkologischen Langzeitergebnisse und die ungenügend strukturierte Dokumentation als Basis für Vergleiche und Transparenz dargestellt. Basis der Analyse zur Versorgung in Deutschland waren wertvolle Ergebnisse der Qualitätssicherungsgruppe Kolon- und Rektumkarzinom des An-Instituts für Qualitätssicherung in der operativen Medizin an der
Universität Magdeburg zum Zusammenhang von chirurgischen Komplikationsraten, Morbidität und Fallzahlen, die 2001 von Marusch et al. $[13,14]$ publiziert wurden. In der zusammenfassenden Wertung wurde von Link et al. [11] der Chirurg im interdisziplinären Team (Tumor-Boards) zum entscheidenden Prognosefaktor deklariert, da er durch seine chirurgische Erfahrung und Spezialisierung in Verbindung mit entsprechenden Krankenhaus- und Teamstrukturen Garant für eine optimale Kurzzeitergebnisqualität und Voraussetzung für eine entsprechende onkologische Langzeitergebnisqualität darstellt. Die Autoren sahen den Chirurgen als Prognosefaktor im multimodalen Konzept mit $>80 \%$ an. Die multimodale Therapie mit entsprechender Qualität der Chemo- und Strahlentherapie sind der wesentliche Faktor für die Beeinflussung der Langzeitprognose [11].

Die aktuelle klinische Versorgungsforschung in Deutschland zur Erfassung der Behandlungssituation von Patienten mit Kolonkarzinom wird durch die multizentrische Beobachtungsstudie „Qualitätssicherung Kolon/Rektum-Karzinome (Primärtumor)“ des An-Instituts zur Qualitätssicherung in der operativen Medizin an der Otto-von-Guericke-Universität Magdeburg präsentiert. Diese Studie stellt ein valides Instrument zur externen Bewertung der Qualität chirurgischer Eingriffe dar und analysiert die Behandlungssituation und die flächendeckende Versorgungsqualität des Kolonkarzinoms in Deutschland [15]. Die Analyse von 31055 operativ behandelten Patienten mit Kolonkarzinom aus 346 deutschen Kliniken im Zeitraum 2000-2004 zeigte, dass im Stadium UICC III, in dem laut Leitlinie die adjuvante Chemotherapie erforderlich ist, diese in der Versorgungssituation bei 72,4\% der Patienten geplant wurde. Das 5-Jahres-Gesamtüberleben betrug $53,8 \%$ [15]. Sieht man die Divergenz der 72,4\% geplanten Chemotherapien im Stadium UICC III in der Versorgungssituation zu den geforderten $80 \%$ der Krebsgesellschaft, so kann man nur indirekt Rückschlüsse aus der Beobachtungsstudie auf Kontraindikationen oder eventuelle Therapieablehnungen ziehen. Das Durchschnittsalter der Patienten dieser Beobachtungsstudie lag bei 69,8 Jahren, wobei der Anteil der Patienten über 80 Jahre 18,6\% betrug. Der Anteil der ASA-III- und -IV-Patienten betrug 40,0\% und 3,7\%. 67,4\% der Patienten hatten kardiovaskuläre Begleiterkrankungen [16].

Die moderne medizinische Entscheidungsfindung wird durch ein evidenzbasiertes Vorgehen bestimmt. Evidenzbasierte Medizin in der klinischen Routine bedeutet die Integration individueller klinischer Expertise mit der bestmöglichen Evidenz aus systematischer Forschung [17]. Bei der Erstellung evidenzbasierter Leitlinien wird die Evidenzgrundlage hierarchisch kategorisiert, wobei Metaanalysen, begründet auf randomisierten klinischen Studien, als höchste Stufe innerhalb der Kategorisierung eingeschätzt werden [18]. Die Evidenzgrade werden in Leitlinien abgebildet und die Stärke der Leitlinienempfehlung lehnt sich an sie an. Dies spiegelt sich auch in der aktuellen S3-Leitlinie „Kolorektales Karzinom“ wider [19].

Die evidenzbasierten Vorgaben der S3-Leitlinie zeigen die Überlegenheit multimodaler Therapiekonzepte bei der Behandlung des kolorektalen Karzinoms. Während die chirurgische Tumorentfernung und in Ausnahmesituationen die endoskopische Abtragung von Kolontumoren früher Stadien unverändert den einzigen kurativen Ansatz darstellen, publizierten Moertel et al. [20] 1995 eine prospektiv-randomisierte Studie mit dem Nachweis, dass eine adjuvante Chemotherapie im Tumorstadium III eine Verbesserung der 5-Jahres-Überlebensrate um 16\% ermöglicht. Somit erreichte die multimodale Therapie einen bedeutenden Stellenwert in der Therapie des Kolonkarzinoms. Die Umsetzung der 
multimodalen und damit interdisziplinären Behandlungskonzepte stellte eine neue Herausforderung für ein evidenzbasiertes Vorgehen im klinischen Alltag dar. Eine intensivierte adjuvante Chemotherapie durch das FOLFOX-4-Protokoll konnte in der MOSAIC-Studie dieses Ergebnis nochmals optimieren mit dem Nachweis vermehrter neurotoxischer Nebenwirkungen [21]. Die neurotoxische Wirkung ist jedoch oft therapielimitierend, sodass in der onkologischen Praxis derzeit dem modifizierten FOLFOX-6Protokoll ohne 2. 5-FU-Bolus der Vorzug gegeben wird [19].

\section{Behandlungsqualität des kolorektalen Karzinoms in Deutschland \\ $\nabla$}

Entscheidende Kriterien der Beurteilung der Behandlungsqualität stellen unter Anderem das rezidivfreie Überleben und die Lokalrezidivrate dar. Die 1. weltweite Analyse des 5-Jahres-Gesamtüberlebens war die CONCORD-Studie, die 2008 von Coleman et al. [22] publiziert wurde. Dabei wurden Tumorerkrankungen von 1990-1994 sowie ihr Follow-up bis 1999 analysiert. Die deutschen Daten wurden aus den Tumorerkrankungen des Saarlands erhoben. Im internationalen Vergleich von 30 Ländern nahm Deutschland dabei mit einem 5-Jahres-Gesamtüberleben beim Kolonkarzinom mit 56,2\% bei den Frauen und 52,0\% bei den Männern Rang 10 und 13 ein. Beim Rektumkarzinom war dies Rang 13 und 15 mit 52,5\% 5-Jahres-Gesamtüberleben bei Frauen und $47,8 \%$ bei Männern. Deutschland war damit im mittleren Drittel aller Auswertungen. Ausgehend von diesen Daten stellt die Initiative zur Bildung von zertifizierten onkologischen Versorgungsstrukturen, die auf der Basis von Leitlinien interdisziplinär und intersektoral zusammenarbeiten, einen wichtigen Schritt dar. Ziel ist eine Steigerung der Qualität in der Betreuung sowohl im Hinblick auf die Ergebnis- als auch die Struktur- und Prozessqualität.

\section{Nationaler Krebsplan 2008 \\ $\nabla$}

Auch auf europäischer Ebene hat die Auswertung der Behandlungsdaten onkologischer Patienten zu einer Reihe von Initiativen geführt. In einer Ratsschlussempfehlung von 2008 hat das europäische Parlament seine Mitgliedsstaaten aufgerufen, nationale Pläne für die Krebsbekämpfung zu erarbeiten und vor allem umzusetzen. Im Zuge dieser europäischen Aufgabenstellung und mit dem Ziel der Abstimmung der Aktivitäten aller an der Krebsbekämpfung Beteiligten und der Unterstützung des zielorientierten Vorgehens wurde am 16.06.2008 der Nationale Krebsplan vorgestellt. Initiiert wurde er vom Bundesministerium für Gesundheit in Zusammenarbeit mit der Deutschen Krebsgesellschaft, der Deutschen Krebshilfe und der Arbeitsgemeinschaft Deutscher Tumorzentren. Die Schwerpunkte des Nationalen Krebsplans umfassen in einer ersten Phase 4 Handlungsfelder. Im Handlungsfeld 2 steht neben der Entwicklung evidenzbasierter Leitlinien und dem flächendeckenden Ausbau klinischer Krebsregister die Weiterentwicklung onkologischer Versorgungsstrukturen und deren Qualitätssicherung im Vordergrund [23]. In der Arbeitsgruppe des Nationalen Krebsplans wurde erstmals eine Definition für zertifizierte onkologische Versorgungsstrukturen bzw. zertifizierte Zentren erarbeitet. Demnach sind zertifizierte Zentren ein „Netz von qualifizierten und gemeinsam zertifizierten, multi- und interdisziplinären, transsektoralen und ggf. standortübergreifenden Einrichtungen (Krankenhäuser, vertragsärzt- liche Versorgung, Rehabilitationseinrichtungen), die, sofern fachlich geboten, möglichst die gesamte Versorgungskette für Betroffene abbilden ..." [24]. Die zertifizierten Netzwerke gliedern sich dabei in 3 Zertifizierungsstufen. Dieses 3-Stufen-Modell setzt sich aus den Organkrebszentren (C), den Onkologischen Zentren (CC) und den Onkologischen Spitzenzentren (CCC) mit Forschungsschwerpunkten zusammen. Organkrebszentren sind Zentren, die auf ein Organ oder ein Fachgebiet spezialisiert sind. Sie stellen die Basisstruktur des 3-Stufen-Modells dar. Ein Onkologisches Zentrum erstreckt sich auf mehrere Organe und Fachgebiete und ein Onkologisches Spitzenzentrum ist ein Onkologisches Zentrum mit Forschungsschwerpunkten [25].

\section{Deutsche Krebsgesellschaft und Zentrumsbildung $\nabla$}

Innerhalb des 3-Stufen-Konzepts werden die Organkrebszentren und die Onkologischen Zentren durch die Deutsche Krebsgesellschaft (DKG) zertifiziert. Die onkologischen Spitzenzentren werden über die Deutsche Krebshilfe (DKH) ausgelobt und gefördert. Die Entwicklung des Zertifizierungssystems begann 2003 mit der Zertifizierung der ersten Brustkrebszentren in Kooperation mit der Deutschen Gesellschaft für Senologie. Die Initiative resultierte aus einer vergleichenden Analyse von Defiziten in den Behandlungsergebnissen Deutschlands zu anderen Ländern [5].

Ziel des Zertifizierungssystems der Deutschen Krebsgesellschaft ist die Verbesserung der Versorgung onkologischer Patienten durch die umfassende Betreuung der Patienten in allen Phasen und für alle Bereiche ihrer Erkrankung, indem die Partner des Netzwerks interdisziplinär, berufsgruppen- und sektorenübergreifend zusammenarbeiten. Das Zertifikat soll dabei dem Patienten und seinen Angehörigen die Transparenz und Sicherheit geben, dass für seine Erkrankung hohe Qualitätsstandards erfüllt werden.

Grundlage für die Zusammenarbeit in einem zertifizierten Zentrum sind die Empfehlungen der aktuellen S3-Leitlinien. Diese Empfehlungen sind in Form von Kennzahlen und Qualitätsindikatoren in die Erhebungsbögen für die Zertifizierung aufgenommen und die Zentren müssen im Rahmen der jährlichen Audits nachweisen, dass sie diese Kennzahlen erfüllen und ihre Patienten dementsprechend leitlinientreu behandeln. Auf diese Weise wird ein weiteres wichtiges Ziel des Zertifizierungssystems erreicht, nämlich die Implementierung evidenzbasierter Leitlinien in den klinischen Alltag [25]. Gerade an dieser Stelle bestehen innerhalb des Gesundheitssystems, ungeachtet der Art der Erkrankung, häufig Kommunikationsdefizite. Leitlinien werden erstellt, die Anwendung und Umsetzung dieser Leitlinien wird aber nicht koordiniert und bleibt dem Engagement Einzelner überlassen. In der Onkologie haben wir die bisher beispiellose und zugleich beispielgebende Situation, dass zwischen dem durch AWMF (Arbeitsgemeinschaft der Wissenschaftlichen Medizinischen Fachgesellschaften), DKG und DKH gegründeten Leitlinienprogramm Onkologie und dem Zertifizierungssystem der Deutschen Krebsgesellschaft und der Deutschen Krebshilfe eine sehr enge und funktionstüchtige Schnittstelle besteht. Sowohl die Erstellung der Qualitätsindikatoren in den Leitlinien als auch die Diskussion der Auswertung dieser Indikatoren erfolgt bidirektional in den Gremien der beiden Institutionen. Somit besteht mit dem Zertifizierungssystem die Chance, zum Einen die Anwendung von Leitlinieninhalten sowohl $\mathrm{zu}$ fordern als auch $\mathrm{zu}$ fördern und gleichzeitig die Effekte der Leitlinienanwendung auf die Ergebnisqualität der Versorgung mittelfristig zu evaluieren. 


\section{Netzwerkbildung des Darmkrebszentrums}

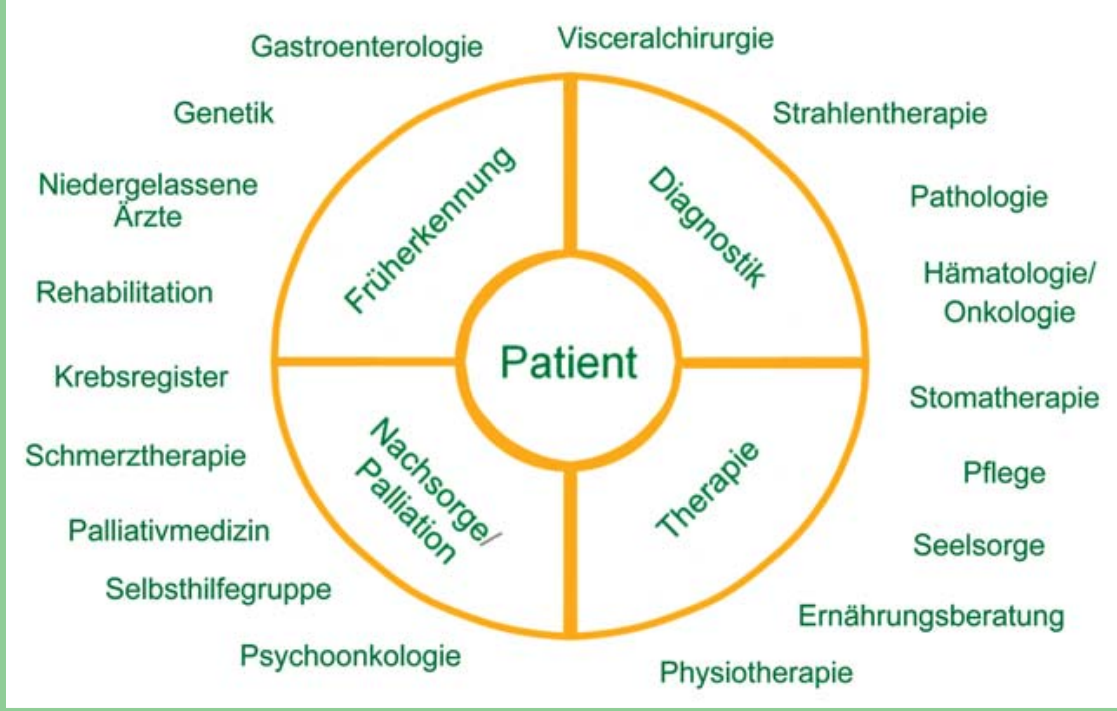

Abb. 1 Netzwerkbildung in einem Darmkrebszentrum: Interdisziplinäre, transsektorale und berufsübergreifende Zusammenarbeit.

\section{Darmkrebszentrum als Modell der onkologischen Versorgung}

Ein Darmkrebszentrum ist im 3-Stufen-Modell der onkologischen Versorgung ein Organkrebszentrum (C). Die ersten Darmkrebszentren wurden 2006 zertifiziert. Zum 31.05.2011 waren durch die Deutsche Krebsgesellschaft 207 Darmzentren zertifiziert und 63 Anfragen bzw. laufende Verfahren lagen vor [26].

Auch wenn aus biologischen und therapeutischen Gründen die Tumorlokalisationen Kolon und Rektum 2 Entitäten darstellen, so wird in der S3-Leitlinie „Kolorektales Karzinom“ und im Prozess der Darmzentrenbildung auf die Versorgung beider Entitäten gleichermaßen gezielt $[11,19]$.

Ein Beispiel für die Notwendigkeit der Gründung von Darmkrebszentren war der Nachweis der Diskrepanz in der Durchsetzung der adjuvanten Chemotherapie in den Kliniken bei Patienten mit einem R0-resezierten Kolonkarzinom im Stadium UICC III. Die Notwendigkeit der adjuvanten Therapie ist mit der Evidenzstärke 1a festgelegt und in zahlreichen randomisierten Studien konnte ein signifikanter Überlebensvorteil der Patienten, welche eine adjuvante Chemotherapie erhalten haben, gezeigt werden [19]. Dennoch ergab eine Auswertung der klinischen Krebsregister über die Arbeitsgemeinschaft Deutscher Tumorzentren e.V. anhand der Daten von annähernd 100000 Patienten, die sowohl in nicht zertifizierten als auch in zertifizierten Kliniken behandelt wurden, dass nur 61,2\% der Patienten diese Therapie erhielten. Die Deutsche Krebsgesellschaft nahm diese Empfehlung der Leitlinie in ihre Kennzahlenabfrage auf und fordert als Sollvorgabe, dass $\geq 80 \%$ Chemotherapien bei Patienten im Stadium UICC III durchgeführt werden. Die erstmalige Analyse der Kennzahlenergebnisse von 158 zertifizierten Standorten aus dem Jahr 2010 zeigt, dass in den zertifizierten Zentren 73,8\% der infrage kommenden Patienten eine adjuvante Chemotherapie erhalten haben. Bei der Betrachtung der beiden Auswertungen fällt auf, wie groß die Unterschiede in der klinischen Versorgung wirklich sind, wenn man bedenkt, dass in den Auswertungen der klinischen Krebsregister auch zertifizierte Zentren berücksichtigt wurden. Das Ergebnis der zertifizierten Darmkrebszentren aus dem Kalenderjahr 2010 kann noch nicht das Ziel auf dem stetigen
Weg der Verbesserung der Versorgung der Patienten mit einem kolorektalen Karzinom sein. Durch die immer wiederkehrende Auswertung und Reflexion der Ergebnisse der Zentren in Form von strukturierten Dialogen während des Audits wird die kritische Auseinandersetzung und im Folgenden die Weiterentwicklung der Zentren nachhaltig unterstützt. Gerade mit dem Kennzahlenbogen steht den Zentren ein hervorragendes Instrument zur Verfügung, um die eigenen Behandlungsergebnisse für alle Partner des Zentrums transparent zu machen, zu prüfen und für die interdisziplinäre Diskussion zu nutzen und bei Notwendigkeit Strukturen und Prozesse innerhalb des Zentrums anzupassen. Darüber hinaus bietet die jährliche Gesamtauswertung der Kennzahlen dem Darmkrebszentrum die Möglichkeit, die eigenen Ergebnisse im Sinne eines Benchmarkings mit den Ergebnissen der anderen zertifizierten Zentren zu vergleichen und wichtige Informationen über die Stärken und Schwächen der eigenen Arbeit in einem bundesweiten Vergleich zu erlangen.

Die Darmkrebszentren reflektieren in ihren Anforderungen die Erkenntnisse der publizierten Analysen zu Qualitätsparametern in der kolorektalen Chirurgie $[11,12]$. So wird durch die Festlegung der operierenden Chirurgen im Darmkrebszentrum der notwendigen chirurgischen Erfahrung und Spezialisierung des Chirurgen als Prognosefaktor im interdisziplinären Team Rechnung getragen.

Die Gründung eines Darmkrebszentrums erfordert die Bereitstellung der für ein Organkrebszentrum erforderlichen Strukturen. Diese sind geprägt durch eine hohe Komplexität der Interdisziplinarität, die sich in einem Netzwerk abbilden ( $\bullet$ Abb. 1).

Die Etablierung eines funktionierenden Darmkrebszentrums basiert auf der Bereitschaft aller Fachdisziplinen und Experten. Das Engagement der treibenden Fachrichtung zielt auf die benachbarten Fachdisziplinen für eine gemeinsame, interdisziplinäre Behandlung. Die geforderte gemeinsame Tumorkonferenz, die auch heute noch kein Standard in vielen Krankenhäusern ist, führt zu einer komplexen Betrachtung der im Darmkrebszentrum behandelten Patienten. Die geforderten Strukturen und Prozesse sind die Basis dafür, dass unsere Patienten auch mit unterstützenden Leistungen von Psychoonkologen, Ernährungstherapeuten, Sozialdienstmitarbeitern, Seelsorgern und Anderen be- 
Tab. 1 Vorteile für die behandelnden Fachbereiche im Darmkrebszentrum.

- Vorteile in der Durchsetzung medizinisch notwendiger Strukturen

(z. B. fachübergreifende Tumorkonferenz)

- Konfliktreduktion in der Zusammenarbeit mit den anderen Fachrichtungen

- ständige Kontrolle der Behandlungsqualität aller Fachbereiche im Darmkrebszentrum, woraus eine Optimierung der Zusammenarbeit resultiert

- fachliche Präsentation in der Öffentlichkeit

- transparente Prozesse für alle Partner des Zentrums

- bessere Verhandlungsposition gegenüber dem Klinikmanagement.

treut werden. Diese Teile der multimodalen Behandlungskette würden ohne die Forderungen an eine Zentrumsbildung nicht immer für die Patienten zur Verfügung stehen. Sicherlich kann eine optimale Behandlung an einzelne Mediziner gebunden sein. Ein Darmkrebszentrum bedarf jedoch einer funktionierenden Gesamtlogistik, in der der einzelne Mediziner ein Baustein ist und in der sich der einzelne Mediziner in ein Gesamtkonzept einreihen muss.

Die Implementierung eines Darmkrebszentrums benötigt personelle, zeitliche, materielle und finanzielle Ressourcen, diese müssen durch den Klinikbetreiber als Vorleistung realisiert werden. Im deutschen DRG-Vergütungssystem finden diese Anstrengungen und Qualitätsmerkmale keinen Niederschlag. In einigen Bundesländern wurde von Organzentren ein finanzieller $\mathrm{Zu}-$ schlag von den Krankenkassen eingefordert, bisher wurde aber dieses Anliegen stets abgelehnt. In der Zukunft müssen solche Qualitätsstrukturen auch finanzielle Anreize haben, daher sollten unsere Fachgesellschaften und die Deutsche Krebsgesellschaft stetig an einer Möglichkeit der Vergütung arbeiten und dies einfordern.

Patienten und ihren Angehörigen wird mit dem Zertifikat eines Darmkrebszentrums eine Entscheidungshilfe für den Ort ihrer Behandlung angeboten. Über das Gütesiegel wird ihnen die Sicherheit gegeben, dass in dem zertifizierten Darmkrebszentrum klar definierte Behandlungspfade sowie kompetente und vertrauensvolle Experten zur Verfügung stehen. Diese sind in der Lage, sie in allen Phasen ihrer Erkrankung kompetent zu begleiten.

Für die behandelnden Fachbereiche im Darmkrebszentrum entstehen aus den Organisationsstrukturen Vorteile, die innovativ die Behandlung und Organisationsprozesse beeinflussen ( Tab. 1). Darmkrebszentren unterliegen einer ständigen strukturellen Weiterentwicklung. Zum Aufgabenfeld gehören die Überprüfung und Aktualisierung von Qualitätsindikatoren und die Reflexion in die Überarbeitung von Leitlinien. Die Notwendigkeit der weiteren Implementierung von Darmkrebszentren in Deutschland wird gemessen werden an einer ständig nachweisbaren Verbesserung der Kurz- und Langzeitergebnisqualität in der Versorgung von Patienten mit kolorektalen Karzinomen.

Interessenkonflikt: Nein

\footnotetext{
Institute

${ }^{1}$ Klinik für Chirurgie,Darmzentrum Treptow-Köpenick, DRK Kliniken Berlin |Köpenick, Berlin, Deutschland

2 Bereich Zertifizierung, Deutsche Krebsgesellschaft e. V., Berlin, Deutschland

3 Otto-von-Guericke-Universität, An-Institut für Qualitätssicherung in der operativen Medizin gGmbH, Magdeburg, Deutschland

${ }^{4}$ Klinik für Chirurgie, DRK Kliniken Berlin|Köpenick, Berlin, Deutschland

${ }^{5}$ Klinik für Allgemein-, Viszeral- und Gefäßchirurgie, Universitätsklinikum Magdeburg A. ö. R., Magdeburg, Deutschland

${ }^{6}$ Klinik für Innere Medizin-Gastroenterologie, Darmzentrum Treptow-Köpenick, DRK Kliniken Berlin |Köpenick, Berlin, Deutschland
}

\section{Literatur}

1 Schalhorn A, Kollig F, Tympner C et al. Kolonkarzinom. In: Sendler A, Hrsg. Manual Gastrointestinale Tumoren des Tumorzentrum München. Empfehlungen zur Diagnostik, Therapie und Nachsorge. 7. Aufl. München: Zuckschwerdt; 2006: 117-160

2 Robert Koch-Institut und die Gesellschaft für epidemiologische Krebsregister in Deutschland e.V. Krebs in Deutschland 2003-2004. Häufigkeiten und Trends. Berlin: RKI; 2008

3 Robert Koch-Institut und die Gesellschaft für epidemiologische Krebsregister in Deutschland e.V. Krebs in Deutschland 2005-2006. Häufigkeiten und Trends. Berlin: RKI; 2010

4 Statistisches Bundesamt. Todesursachen in Deutschland. Gestorbene in Deutschland an ausgewählten Todesursachen 2005. Wiesbaden: Statistisches Bundesamt; 2007

5 Hohenberger W, Stirkat F, Bruns J et al. Krebsregister und Zentrumsbildung. Onkologe 2011; 2: 135-142

6 Siess M. Qualitätsmanagement in der Chirurgie - quo vadis? Chirurg 2003; 74: 499-500

7 Codman E.A. A study in hospital efficiency by case report of the five years of a privat hospital. Boston: Thomas Todd Company; 1917

8 Neuhauser D. Ernest Amory Codmann MD. Qual Saf Health Care 2002; 11: 104-105

9 Passaro E jr., Organ CH jr. Ernest A. Codman: the improper Bostonian. Bull Am Coll Surg 1999; 84: 16-22

10 Schyve PM. The evolution of external quality evaluation: observations from the Joint Commission on Accreditation of Healthcare Organizations. Int J Qual Health Care 2000; 12: 255-258

11 Link KH, Kornmann M, Mann M. Multimodale Therapie von Kolon- und Rektumkarzinom: Qualitätsparameter. Viszeralmedizin 2009; 25: 105-117

12 Bittner R, Burghardt J, Gross E. Qualitätsindikatoren bei Diagnostik und Therapie des Rektumkarzinoms. Zentralbl Chir 2007; 132: 85-94

13 Marusch F, Koch A, Schmidt U et al. Effect of caseload on the short-term outcome of colon surgery: results of a multicenter study. Int J Colorectal Dis 2001; 16: 362-369

14 Marusch F, Koch A, Schmidt $U$ et al. Hospital caseload and the results achieved in patients with rectal cancer. Br J Surg 2001; 88: 1397-1402

15 Kube R, Gastinger I, Mroczkowski P et al. The care of patients with colon cancer: current treatment, and evaluation of new surgical approaches. Dtsch Arztebl Int 2011; 108: 41-46

16 Kube $R$. Die aktuelle chirurgische Therapie des Kolonkarzinoms in Deutschland. Klinische Versorgungsforschung anhand der prospektiven multizentrischen Beobachtungsstudie: Qualitätssicherung Kolon/ Rektum-Karzinom (Primärtumor). Habilitation. Magdeburg: Universität Magdeburg; 2009

17 Sackett DL, Rosenberg WM, Gray JA et al. Evidence based medicine: what it is and what it isn't. BMJ 1996; 312: 71-72

18 Oxford Centre for Evidence-Based Medicine (CEBM). Levels for Evidence (2009). Im Internet: www.cebm.net; Stand: 28.4.2009

19 Schmiegel W, Reinacher-Schick A, Arnold D et al. S3-Leitlinie „Kolorektales Karzinom“ - Aktualisierung 2008. Ergebnis einer evidenzbasierten Konsensuskonferenz (8.-9. Juni 2007). Z Gastroenterol 2008; 46: 799840

20 Moertel CG, Fleming TR, Macdonald JS et al. Fluorouracil plus levamisole as effective adjuvant therapy after resection of stage III colon carcinoma: a final report. Ann Intern Med 1995; 122: 321-326

21 Andre T, Tournigand C, Achille E et al. [Adjuvant treatment of colon cancer MOSAIC study's main results]. Bull Cancer 2006; 93 (Suppl. 1): S5S9

22 Coleman MP, Quaresma M, Berrino F et al. Cancer survival in five continents: a worldwide population-based study (CONCORD). Lancet Oncol 2008; 9: 730-756

23 Bundesministerium für Gesundheit. Nationaler Krebsplan. Im Internet: http://www.bmg.bund.de/glossarbegriffe/nationaler-krebsplan.html 2010; Stand: Februar 2010

24 Bundesministerium für Gesundheit. Nationaler Krebsplan. Im Internet: http://www.bundesgesundheitsministerium.de/fileadmin/dateien/ Downloads/N/Nationaler_Krebsplan/Zielpapier5_Nationaler_Krebsplan_onkologische_Versorgungsstrukturen_110623.pdf. 2011; Stand: 8. Juni 2011.

25 Wesselmann S. Zertifizierte Zentren - sektorenübergreifende Netzwerke der onkologischen Versorgung. Forum 2010; 1: 15-16

26 Deutsche Krebsgesellschaft (DKG). Zertifizierte Darmzentren (Stand 5/ 2011). Im Internet: www.krebsgesellschaft.de; Stand: 31.5.2011 